\title{
Clinico-radiological and functional outcome of Mayo type IIA olecranon fractures managed with tension band wiring in a tertiary care centre in North India
}

\author{
Pankaj Spolia*, Abdul Ghani, Sakib Arfee
}

Department of Orthopaedics, GMC, Jammu and Kashmir, India

Received: 01 September 2021

Accepted: 21 September 2021

*Correspondence:

Dr. Pankaj Spolia,

E-mail: pankajspolia13@gmail.com

Copyright: ( $)$ the author(s), publisher and licensee Medip Academy. This is an open-access article distributed under the terms of the Creative Commons Attribution Non-Commercial License, which permits unrestricted non-commercial use, distribution, and reproduction in any medium, provided the original work is properly cited.

\begin{abstract}
Background: Tension band wiring is the most common operative technique for the internal fixation of olecranon fractures. Stable internal fixation with figure of eight tension band wiring used for simple transverse fractures allows early range of motion, minimize stiffness and gives good results. The aim of this study is to evaluate the functional outcome of simple transverse fractures managed by tension band wiring.

Methods: This was an observational prospective study of 24 patients with Mayo type IIA fractures aged between 18 to 65 years, with mean age of 42.5 years treated by tension band wiring. Functional outcome was assessed with Mayo Elbow Performance Score (MEPS) and Visual Analogue Scale (VAS) subjective pain score.

Results: Our study was conducted on 24 patients with Mayo type IIA, out of which 16 were males and 8 were females. Most common mode of injury was fall from standing height (75\%), followed by road traffic accident (16.7\%) and assault $(8.3 \%)$. The age range was between 18 to 65 years, with mean age of 42.5 years.

Conclusions: Tension band wiring is an effective method for the treatment of transverse, non-comminuted and unstable fractures of the olecranon which provides stable fixation, early rehabilitation and gives excellent results when done in expert hands.
\end{abstract}

Keywords: Tension band wiring, Mayo elbow performance score, Mayo classification

\section{INTRODUCTION}

Olecranon fractures constitute about $10 \%$ of the fractures around elbow in adults. ${ }^{1}$ The incidence of olecranon fractures is increasing in the elderly. ${ }^{2,3}$ Operative treatment of displaced, transverse, non-comminuted fracture of olecranon is associated with good elbow function. ${ }^{3,4}$ Fractures of olecranon are intraarticular and require good anatomic reduction to restore articular congruity and elbow stability. Conservative treatment could provide a good functional outcome in the elderly with low complications. ${ }^{5,6}$ The principle of tension band wiring is to convert the distracting forces on the outer cortex of the ulna during flexion into compressive forces on the articular surface of the olecranon at the fracture site. ${ }^{4}$ Locking plates are suited best to the management of complex olecranon fractures. ${ }^{7}$ The Mayo classification guides the treatment on the basis of displacement and ulnohumeral joint stability: Type I, non- displaced fractures, treated non-operatively, type II are displaced, stable and require surgical treatment, type III are displaced and unstable, need surgical treatment. ${ }^{8}$ Each type has subtypes, $A=$ non comminuted and $\mathrm{B}=$ comminuted. The most common complications in the patients after tension band wiring are pain and hardware symptoms due to migration of $\mathrm{K}$ wires. ${ }^{8,9}$ 


\section{METHODS}

This was an observational prospective study of 24 patients $(n=24)$, satisfying the inclusion criteria, treated with open reduction and internal fixation with tension band wiring from January 2019 to July 2021 at Post Graduate Department of Orthopaedics, Government Medical College, Jammu. Patients were included in the study after proper history, clinical examination, written informed consent for surgery and anaesthesia, and explaining the complications before the surgery. Functional outcome was assessed by Mayo Elbow Performance Score, at 6 month follow-up, consisting of four parameters: pain (maximum score $=45$ points), range of motion (maximum score $=20$ points), stability (maximum score $=10$ points), function $(5$ items, 5 points for each). ${ }^{22}$ Maximum score can be 100 , and the results are graded as excellent if score $>90$; good if score of 75-89; fair if score of 60-74 and poor for score $<6010$. Pain was evaluated using Visual Analogue Scale. ${ }^{11}$

\section{Inclusion criteria}

Isolated olecranon fracture, Mayo type IIA fractures. Age, 18- 65 years. Closed fractures. Duration of trauma less than 2 weeks.

\section{Exclusion criteria}

Olecranon fractures with fracture dislocation and terrible triad injuries, compound fractures, fractures presenting after 2 weeks, polytrauma patients.

\section{Investigations}

X-rays with anteroposterior and lateral views, $\mathrm{CT}$ scan was also done in doubtful and complex fractures. All baseline investigations were done prior to surgery. Informed written consent was taken and antibiotic prophylaxis was given.

\section{Operative technique}

The patient was placed in supine position. A posterior midline incision was made, starting over olecranon tip extended proximally and distally. After fracture reduction, two parallel $1.8 \mathrm{~mm}$ Kirschner wires were placed in the ulna from olecranon tip proximally to distal aspect across the fracture, to engage in the anterior cortex of the proximal shaft of ulna. A transverse tunnel was made with Kirschner wire about $4 \mathrm{~cm}$ distal to the fracture site. 18 guage stainless steel wire was placed in the figure of eight configuration, which was tensioned to reduce, fix and stabilize the fracture fragments.

The parallel K-wires achieve the best force transmission across the fracture. ${ }^{12}$ Long K-wires may cause vascular and nerve injuries. ${ }^{13,14}$ Transcortical K-wires provide higher stability and reduce the risk of wire migration. ${ }^{15}$ Two wire knots provide greater stability than a single knot. ${ }^{16,17}$

\section{Post-operative treatment}

The limb was elevated and placed in above elbow slab for 2-3 days. Post-operative intravenous antibiotics and analgesics were given. Immediate postoperatively, check $\mathrm{X}$-rays were done. Early range of motion exercises was started at 3 rd post-operative day. Sutures were removed at 14th post operative day.

\section{Follow up}

The patients were followed up to 6 months to 1 year, and were assessed clinically and radiologically at 2 weeks, 6 weeks, 3 months, 6 months, 12 months.

At 6 months, patients were assessed by MEPS.

\section{Statistical tools}

As this study was not a comparative study, simple statistical methods of mean and percentage were used.

\section{RESULTS}

The total number of patients presented with olecranon fractures was 33, out of which 24 patients $(72.7 \%)$ had Mayo type IIA fractures, followed by 5 patients $(15.2 \%)$ which had Mayo type IIB fractures, 3 patients $(9.1 \%)$ had Type IIIA fractures and 1 patient (3\%) had Type IIIB fracture. Our study was conducted on 24 patients with Mayo type IIA, out of which 16 were males and 8 were females. Most common mode of injury was fall from standing height $(75 \%)$, followed by road traffic accident $(16.7 \%)$ and assault $(8.3 \%)$.

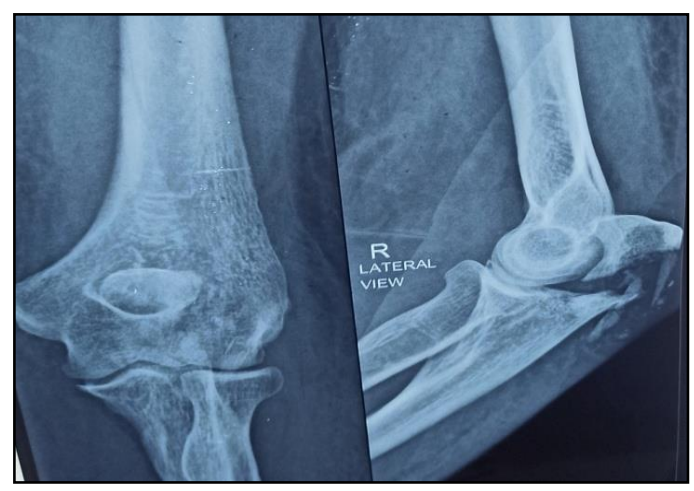

Figure 1: Preoperative X-rays.

The age range was between 18 to 65 years, with mean age of 42.5 years. Majority of the patients had trauma on the right side (right side $=18$, left side $=6$ ). 15 patients were males, 9 were females. Out of 24 patients, 15 patients reported on the same day, 5 patients reported on third day of injury, 4 patients reported after 1 week of injury.

Out of 24 patients, 20 patients were operated within 1 week of trauma and 4 patients were operated within 10 days of 
trauma. Mean operating time was 50 minutes (range $=35$ to 60 minutes).

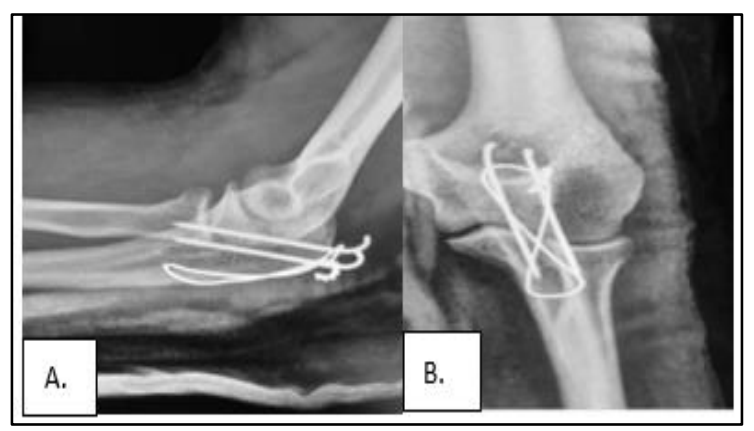

Figure 2: Postoperative x-ray.

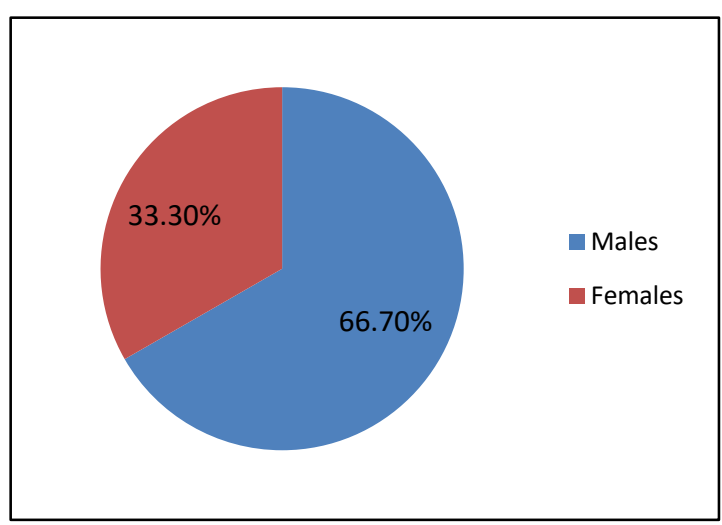

Figure 3: Sex distribution.

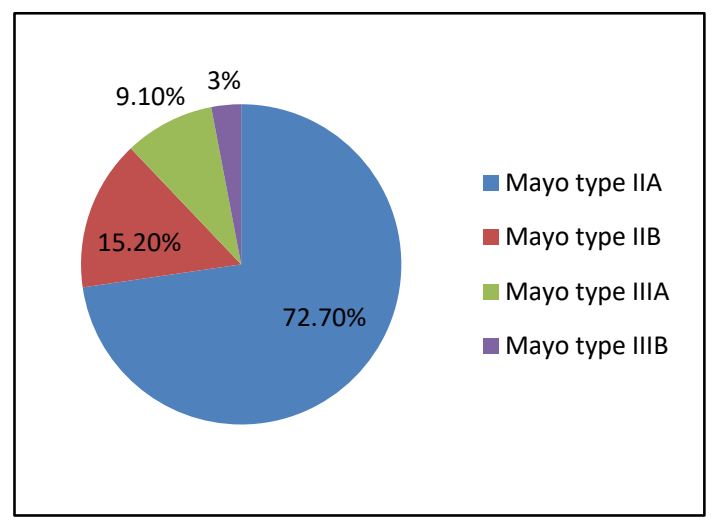

Figure 4: Type of fractures distribution.

\section{Clinico-radiological consolidation}

Clinical union was achieved when there was no pain and tenderness at the fracture site and there was no abnormal mobility in two planes. Radiological union was achieved when the fracture line was obscured and there was atleast three cortex continuity along with bridging bony trabeculae. Out of 24 patients, 20 patients had clinical union at 7.5 weeks and 4 achieved clinical union at 9.2 weeks, with an average healing at 8.5 weeks (range, 6 weeks to 10 weeks).

In 20 patients, radiological union was achieved at 11.8 weeks and in 4 patients the radiological union was achieved at 12.9 weeks, with an average radiological union at 12.2 weeks (range, 9 weeks to 13 weeks).

Table 1: Comparison of radiological union.

\begin{tabular}{|c|c|}
\hline Study & Average time of union \\
\hline Hume et $\mathrm{al}^{24}$ & 14 weeks \\
\hline Fan et al $^{25}$ & 14 weeks \\
\hline Macko et al ${ }^{9}$ & 12 weeks \\
\hline Present study & 12.2 weeks \\
\hline
\end{tabular}

Table 2: Comparison of functional outcome.

\begin{tabular}{|lllll|}
\hline Study & Results in percentage & \\
\hline $\begin{array}{l}\text { Murphy et } \\
\text { ald }^{\mathbf{2 8}}\end{array}$ & 60 & 10 & 30 & - \\
\hline $\begin{array}{l}\text { Yusufbhai } \\
\text { et al }^{\mathbf{2 7}}\end{array}$ & 72 & 16 & 12 & - \\
\hline Xieyuan $^{\mathbf{2 9}}$ & 53.33 & 40 & 6.66 & - \\
\hline $\begin{array}{l}\text { Present } \\
\text { study }\end{array}$ & 79.16 & 12.5 & 4.17 & 4.17 \\
\hline
\end{tabular}

\section{Functional evaluation}

Patients were functionally evaluated on the basis of MEPS at 6 months. The mean flexion was 132.4 degree (range $=$ 90 to 140), mean extension loss was 8.2 degrees (range $=$ 5 to 9.5 degrees), mean arc motion was 124.2 degree. The average MEPS was 91.2 (range, 55 to 100). In the present study, at 6 month follow up, 20 patients had no pain, 4 patients had pain at the extremes of motion.

Out of 24 patients, $(n=19,79.16 \%)$ had excellent outcome, $(n=3,12.5 \%)$ had good outcome, $(n=1,4.17 \%)$ had fair outcome, $(n=1,4.17 \%)$ had poor outcome. Superficial wound infection was seen in $n=1,4.17 \%$ of patients which was managed by wound wash and antibiotics.

The main postoperative complaints were the hardware symptoms due to proximal migration of $\mathrm{K}$-wires causing pain reported in $4(16.67 \%)$ patients.

Table 3: Comparison of postoperative complications.

\begin{tabular}{|lllll|}
\hline Complication & Ashif et al $^{27}$ & Deepak et al $^{30}$ & Murphy et al $^{28}$ & Present study \\
\hline Superficial infection & $\mathbf{N}(\%)$ & $\mathbf{N}(\%)$ & $\mathbf{N}(\%)$ & $\mathbf{N}(\%)$ \\
\hline Hardware symptoms & $3(12)$ & $2(6.66)$ & - & $1(4.17)$ \\
\hline
\end{tabular}




\section{DISCUSSION}

Olecranon fractures are caused by direct injury to the posterior part of the elbow joint or due to triceps pull because of fall on partially flexed elbow. ${ }^{18}$ In the present study, incidence of fracture was higher in men upto the 4th decade, followed by elderly women above 60 years of age.

In the present study, incidence of olecranon fractures was greater for males upto 4 th decade $(n=16,66.7 \%)$ and $n=8$, $33.3 \%$ were females. Rommens et al, Femke et al and Sullivan et al in their study also reported the higher incidence of olecranon fractures in males in younger males. ${ }^{19-21}$

In the present study, most common fractures seen were Mayo IIA patients $(72.7 \%)$ followed by Mayo type IIB fractures (15.2\%), Type IIIA fractures $(9.1 \%)$ and Type IIIB fractures (3\%). Similar results were reported by Marco et al, Chalidis et al3 and Bruggemann et al. ${ }^{22,23}$

The radiological union was achieved at an average time of 12.2 weeks (range, 9 weeks to 13 weeks). Similar results were reported by Hume et al, Fan et al and Macko et al. ${ }^{9,24,25}$

In the present study, the functional assessment was assessed by MEPS. The mean flexion was 132.4 degree (range $=90$ to 140), mean extension loss was 8.2 degrees (range $=5$ to 9.5 degrees), mean arc motion was 124.2 degree. Mean supination was 79.2 degrees. Mean pronation was 78.1 degrees). Similar results were reported by Anani et al. ${ }^{26}$ The average MEPS was 91.2 (range, 55 to 100$)$. Out of 24 patients, $(\mathrm{n}=19,79.16 \%)$ had excellent outcome, $(n=3,12.5 \%)$ had good outcome, $(n=1,4.17 \%)$ had fair outcome, $(n=1,4.17 \%)$ had poor outcome. Similar results were reported by Chalidis et al, Yusufbhai et al. ${ }^{3,27}$

In the present study, hardware symptoms were reported in $16.67 \%$ patients. In the present study, the results were comparable with the literature.

\section{CONCLUSION}

Tension band wiring is an effective method for the treatment of transverse, non comminuted and unstable fractures of the olecranon which provides stable fixation, early rehabilitation and gives excellent results when done in expert hands.

Funding: No funding sources

Conflict of interest: None declared

Ethical approval: The study was approved by the institutional ethics committee

\section{REFERENCES}

1. Veillett CJH, Steinmann SP. Olecranon fractures. Orthop Clin North Am. 2008;39:229-36.
2. Duckworth AD, Court-Brown CM, McQueen MM. Isolated displaced olecranon fracture. J Hand Surg (Am) 2012;37A:341-5.

3. Chalidis BE, Sachinis NC, Samoladas EP, Dimitriou CG, Pournaras JD. Is tension band wiring technique the 'gold standard' for the treatment of olecranon fractures? A long term functional outcome study. J Orthop Surg Res. 2008;3:9

4. Schneider MM, Nowak TE, Bastian L, Katthagen JC, Isenberg J, Rommens PM et al. Tension band wiring in olecranon fractures: the myth of technical simplicity and osteosynthetical perfection. Int Orthop. 2014;38:847-55.

5. Duckworth AD, Bugler KE, Clement ND. Nonoperative management of displaced olecranon fractures in low-demand elderly patients. J Bone Joint Surg Am. 2014;96(1):67-72.

6. Gallucci GL, Piuzzi NS, Slullitel PAI. Non-surgical functional treatment for displaced olecranon fractures in the elderly. Bone Joint J. 2014;96$\mathrm{B}(4): 530-34$.

7. Kloen P, Buijze GA. Treatment of proximal ulna and olecranon fractures by dorsal plating. Oper Orthop Traumatol. 2009;21:571-85.

8. Helm RH, Miller SW. The complications of surgical treatment of displaced fractures of the olecranon. Injury. 1987;18(1):48-50.

9. Macko D, Szabo RM. Complications of tension-band wiring of olecranon fractures. J Bone Joint Surg Am. 1985;67:1396-401.

10. Morrey BF, An KN, Chao EYS. Functional evaluation of the elbow. In: The Elbow and Its Disorders. 2nd edition. Edited by Morrey BF. Philadelphia: WB Saunders. 1993;86-9.

11. Jensen MP. Interpretation of visual analogue scale ratings and change scores. JPain. 2003;407-14.

12. Hak DJ, Stewart RL. Prinzip der Zuggurtung. In: AO-Prinzipien des Frakturmanagements. Auflage. Rüedi TP, Buckley RE, Moran CG (eds). Georg Thieme Verlag KG, Stuttgart. 2008;2:249-54.

13. Parker JR, Conroy J, Campbell DA. Anterior interosseus nerve injury following tension band wiring of the olecranon. Injury. 2005;36(10):1252.

14. Thumroj E, Jianmongkol S, Thammaroj J. Median nerve palsy after operative treatment of olecranon fracture. J Med Assoc Thai. 2005;88(10):1434-7.

15. Van der Linden SC, van Kampen A, Jaarsma RL. Kwire position in tension-band wiring technique affects stability of wires and long-term outcome in surgical treatment of olecranon fractures. Journal of shoulder and elbow surgery/American Shoulder and Elbow Surgeons. 2012;21:405-11.

16. Deliyannis SN. Comminuted fractures of the olecranon treated by the Weber-Vasey technique. Injury. 1973;5(1):19-24.

17. Mauffrey C, Krikler S. Surgical techniques: how I do it? Open reduction and tension band wiring of olecranon fractures. Injury. 2009;40(4):461-5.

18. Horner SR, Sadasivan KK, Lipka JM, Saha S. Analysis of mechanical factors affecting fixation of 
olecranon fractures. Orthopedics. 1989;12(11):1469725.

19. Rommens PM, Kuchle R, Schneider RU, Reuter M. Olecranon fractures in adults: factors influencing outcome. Injury. 2004;35:1149-57.

20. Claessen FMAP, van den Bekerom MPJ, van Dijk CN, Goslings JC, Kerkhoffs GMMJ, Doornberg JN, et al. Shoulder elbow platform. Tension band wiring for simple olecranon fractures: evaluation of surgical technique. J Orthop Traumatol. 2017;18(3):275-81.

21. Connor W. Sullivan and Khusboo Desai. Classifications in brief: Mayo Classification of olecranon fractures. Clin Orthop Relat Res. 2019;477(4):908-10.

22. Morrey BF, Adams RA. Semiconstrained arthroplasty for the treatment of rheumatoid arthritis of the elbow. J Bone Joint Surg (Am). 1992;74:47990.

23. Bruggemann A, Mukka S, Wolf O. Swedish fracture register. Eur J Trauma Emerg Surg. 2021.

24. Hume MC, Wiss DA, Olecranon fractures. Clinical and radiographic comparison of tension band wiring and plate fixation. Clin Orthop Relat Res. 1992;285:229-35.
25. Fan $\mathrm{G}, \mathrm{Wu} \mathrm{CC}$, Shin $\mathrm{CH}$. Olecranon fractures with tension band wiring techniques - comparison among three different configurations. Chang Gung Medical Journal. 1993;16:231-8.

26. Anani A. The Annals Of African Surgery. 2011;8.

27. Suthar AY. International Journal of Health and Clinical Research. 2020;3(6):26-35.

28. Murphy DF, Greene WB, Damerson TB. Displaced olecranon fractures in adults. Clinical evaluation. Clin Orthop. 1987;224:215-23.

29. Xieyuan J. Operative treatment of olecranon fracture associated with anterior dislocation of the elbow. Chinese J of Orthop. 2000;20(3):154-6.

30. Aher DK, Pandey SK, Alawa S, Pathak A. Evaluation of functional outcome of tension band wiring in olecranon fractures and factors effecting the overall functional outcome. Int J Orthop Sci. 2018;4(2):3346.

Cite this article as: Spolia $\mathrm{P}$, Ghani $\mathrm{A}$, Arfee $\mathrm{S}$. Clinico-radiological and functional outcome of Mayo type IIA olecranon fractures managed with tension band wiring in a tertiary care centre in North India. Int J Res Orthop 2021;7:1099-103. 